\title{
REVISIONES
}

\section{El Enfoque Bilingüe en la Educación de Sordos: sus implicancias para la enseñanza y aprendizaje de la lengua escrita*}

\author{
The Bilingual Approach to Deaf Education: implications \\ for teaching and learning written language
}
O Enfoque Bilíngue na Educação de Surdos: implicações para o ensino e aprendiza- gem da língua escrita

\author{
María Rosa Lissi, ${ }^{\text {a }}$ Kristina Svartholm, ${ }^{\mathrm{b}}$ Maribel González ${ }^{\mathrm{c}}$ \\ aPontificia Universidad Católica de Chile, Escuela de Psicología. Telf.: 5623544852. \\ Correo electrónico: mlissi@uc.cl \\ ${ }^{b}$ Universidad de Estocolmo, Departamento de Lingüística. Telf.: 468162000. \\ Correo electrónico: kristina.svartholm@ling.su.se \\ cPontificia Universidad Católica de Chile, Escuela de Psicología. Telf.: 5623544852. \\ Correo electrónico: mdgonza2@uc.cl
}

\begin{abstract}
RESUMEN
En este artículo se revisan algunos antecedentes sobre el enfoque bilingüe en la educación de sordos, se describe específicamente cómo ha sido implementado en Suecia, y se hace referencia a la incorporación de este enfoque en la educación de sordos en Chile. Posteriormente, se analiza la forma en que la Lengua de Señas puede ser usada para enseñar la lengua escrita en contextos educativos bilingües, a través del análisis de ejemplos tomados de clases para niños de primer ciclo básico en una escuela de sordos. Finalmente, se enfatizan las implicancias del modelo bilingüe para el proceso de enseñanza-aprendizaje de la lengua escrita en la educación de sordos y se discuten algunas consideraciones para seguir avanzando en este ámbito en el contexto educacional chileno.
\end{abstract}

Palabras clave: sordos, educación bilingüe, lengua escrita, lengua de señas.

\begin{abstract}
This article reviews the background of the bilingual approach in deaf education, it describes more specifically how it has been implemented in Sweden, and it refers to the incorporation of this approach in deaf education in Chile. Further on, it analyzes the way in which Sign Language can be used to teach written language in bilingual education contexts, using examples from primary education classes for children at a school for the deaf. Finally the article emphasizes the implications of the bilingual model for the process of teaching and learning written language in deaf education, and it discusses some considerations for moving forward in this area in the Chilean education context.
\end{abstract}

Key words: deaf, bilingual education, written language, sign language.

\section{RESUMO}

Revisam-se alguns antecedentes sobre o enfoque bilíngue na educação de surdos, descreve-se, mais especificamente, como foi sua implementação na Suécia e faz-se referência à incorporação de este enfoque à educação de surdos no Chile. A seguir, analisa-se a forma em que a Língua de Sinais pode ser usada para ensinar a língua escrita em contextos educativos bilíngues, por meio de análises de exemplos retirados de aulas para crianças de primeiro ano do Ensino Fundamental em uma escola para surdos. Finalmente, enfatizam-se as implicações do modelo bilíngue para o processo

* $\quad$ Este trabajo se realizó con financiamiento de Conicyt, a través del proyecto FONDECYT No 1060762 "LS y lengua escrita en niños sordos. Su adquisición y posibles interacciones en un contexto educativo bilingüe" y los proyectos de Incentivo a la Cooperación Internacional № 7080110 y No 7060150. 
ensino-aprendizagem da língua escrita na educação de surdos e discutem-se algumas considerações para continuar com os avanços nesse âmbito, no contexto educacional chileno.

Palavras chave: surdos, educação bilíngue, língua escrita, língua de sinais.

\section{INTRODUCCIÓN}

La educación de sordos se ha caracterizado históricamente por la controversia entre distintos enfoques, básicamente por la oposición entre aquellos que enfatizan el desarrollo de la lengua oral, descartando el uso de la lengua de señas (en adelante LS) utilizada por la comunidad sorda del país y aquellos que defienden un enfoque en que ésta se reconoce como la primera lengua de las personas sordas, utilizándola para enseñar contenidos y desarrollando el conocimiento de la lengua mayoritaria como una segunda lengua. En este trabajo, se entregan antecedentes que fundamentan la adopción de un modelo bilingüe en la educación de sordos y se analiza cómo, desde este enfoque, pueden implementarse estrategias en las que la LS sea utilizada para desarrollar la lengua escrita.

\subsection{EDUCACIÓN DE SORDOS Y LITERACIDAD: LAS LIMITACIONES DEL MODELO ORALISTA}

En términos generales, la educación de sordos no ha sido muy exitosa en la enseñanza de la lectura y la escritura a los niños sordos. Los resultados reportados consistentemente en EE.UU. y otros países muestran que el logro académico, incluyendo el nivel lector de los jóvenes sordos que egresan del sistema escolar, es equivalente a lo que es considerado normal en niños oyentes de tercero o cuarto básico en promedio (Power y Leight, 2000; Wilbur, 2000).

A pesar de décadas de esfuerzo concertado, la mayoría de los niños sordos progresan solo a una fracción de la tasa en que lo hacen los niños oyentes en lo que respecta al aprendizaje de la lectura /..../ Solo alrededor del 3 por ciento de aquellos que tienen 18 años leen al mismo nivel que lo hace un lector promedio de 18 años (Marschark, Lang y Albertini, 2002: 157).

Resultados similares fueron reportados desde Suecia durante los 1960 y los 1970. A pesar del rápido y extenso desarrollo tecnológico a partir de 1950, el que trajo como resultado mejores audífonos y equipamiento en la sala de clases de las escuelas, la habilidad promedio para leer sueco de un joven sordo egresado del sistema escolar, estaba muy por debajo del nivel apropiado para su edad, al igual que sus habilidades de lenguaje oral (Amcoff, 1973; Nordén 1969; Svartholm, 1984). La principal explicación de por qué el aprendizaje de un lenguaje hablado constituye una dificultad tan grande para los niños sordos, es que para estos el habla no está asociada primordialmente a sonidos, sino sólo a pequeños movimientos de la boca. Estos movimientos que el niño puede ver son muy similares unos de otros y, por tanto, muy difíciles de descifrar. A pesar de esto, la educación de sordos ha insistido en muchos casos en usar el lenguaje oral como la base para todo el aprendizaje en las clases. Esto es lo que se conoce como el método oral; la filosofía educacional que está a su base es el "oralismo" (Moores, 1987).

Aunque el lenguaje hablado era el foco principal en el método oral, en la enseñanza de la lectura y la escritura los profesores recurrían también a la imitación, i.e., hacían que los niños copiaran palabras y oraciones del pizarrón. Para muchos niños sordos, estas actividades resultaban carentes de significado y no lograban desarrollar competencias en lectoescritura a partir de estas (Lynas, 2005; Svartholm, 1984). 


\subsection{LA COMUNICACIÓN TOTAL COMO ALTERNATIVA AL ORALISMO}

Hasta finales de los 70 el lenguaje hablado era, en la mayoría de los países, la única modalidad de comunicación aceptada en las aulas para niños sordos (Moores, 1987; Power y Leigh, 2000). Uno de los argumentos encontrados en la literatura es que, al comunicarse con señas, el niño sordo nunca experimentaría la motivación necesaria para enfrentar la difícil tarea de aprender a hablar. Más aún, se ha argumentado que las señas son concretas, manteniendo al niño en un bajo nivel de desarrollo cognitivo e impidiéndole comunicar ideas abstractas. La investigación lingüística acerca de las LS ha probado que estas concepciones son erróneas; pero siguen siendo encontradas entre muchos educadores de sordos (Svartholm, 1984).

A finales de 1960 y principios de la década de los 70 la necesidad de un cambio en los métodos para la enseñanza de los sordos se hizo evidente en los EE.UU. y también en otros países, particularmente en Suecia. Para entonces, el concepto de Comunicación Total se había difundido. En la práctica, Comunicación Total significa que los profesores pueden utilizar señas o gestos simultáneamente con el habla y cualquier otro medio visual para transmitir un mensaje. Así, el niño sordo no tiene que depender sólo del habla para comprender lo que se dice en clases (Moores, 1987).

Algo más tarde, en EE.UU. varios sistemas artificiales, correspondientes a distintas versiones del "Inglés Signado", fueron desarrollados por educadores de sordos con propósitos pedagógicos y se suponía que presentarían los elementos del inglés hablado en señas. Un sistema similar, denominado "Sueco Signado", fue construido en Suecia en la década de los 70. El postulado fundamental, ligado al uso de estos sistemas, era que las palabras del lenguaje hablado y las señas debían usarse simultáneamente; de este modo, a cada palabra en el lenguaje hablado le correspondería una seña. Se pensaba que este método no solo haría la comunicación entre personas sordas y oyentes más fácil, sino que también haría más fácil para los niños sordos el aprender a hablar el idioma del país (Bergman, 1977). Sin embargo, después de aproximadamente 25 años del uso pedagógico de sistemas de este tipo en EE.UU., el promedio de estudiantes sordos de secundaria había logrado avanzar tan solo un año en su desarrollo: de tercero a cuarto grado (Paul y Quigley, 1994). En Suecia los primeros años del uso del sueco signado fueron bastante prometedores. Sin embargo, resultó que las mejoras en la comunicación del aula fueron pequeñas y poco satisfactorias, al igual que las mejoras en las habilidades en el uso del sueco en los niños sordos (Ahlgren 1984; Svartholm 1997). Los niños sordos no percibieron el sueco signado como sueco, sino más bien como señas acompañadas de movimientos de la boca, por lo que no se trataba de una estrategia eficiente para el aprendizaje de dicha lengua (Svartholm, 2010).

\subsection{RECONOCIMIENTO DEL ROL DE LA LS: LA PROPUESTA DE EDUCACIÓN BILINGÜE PARA LOS SORDOS}

La explicación de por qué el uso simultáneo del habla y las señas no funcionó como se esperaba vino después, con el conocimiento obtenido a través del estudio de las lenguas naturales de señas, las LS utilizadas en las comunidades de sordos en los diferentes países. Estas LS no son internacionales, sino que se han desarrollado al igual que las lenguas habladas, en distintos contextos culturales, y constituyen sistemas lingüísticos 
muy complejos, cada uno con un léxico diferente y diferentes reglas gramaticales para combinar las señas en las oraciones. Por otra parte, cada seña no es necesariamente equivalente a una palabra en una lengua hablada, ya que debido a características específicas de su uso en el discurso, es posible incluir gran cantidad de información en una sola seña (Schembrie, 2003). Las LS no sólo se producen de forma manual, sino que también utilizan medios no manuales, tales como expresiones faciales, movimientos de la boca, la dirección de la mirada, etc. Todos estos medios contienen diferentes tipos de información lingüística y son utilizados para expresar partes importantes de la gramática. Esto permite producir simultáneamente un grado significativamente mayor de información lingüística, en comparación a los idiomas hablados (Bergman, 1979, 1982). Además, las LS nativas utilizan el espacio tridimensional enfrente del señante y presentan una organización lingüística diferente a la de las lenguas habladas en lo que respecta a la gramática (Bergman 1990; Liddell, 2003).

En resumen, la estructura de la LS difiere en gran medida a la de las lenguas habladas, debido a las diferencias en la producción y la percepción de los dos tipos de lenguajes. Estas diferentes organizaciones explican el por qué la LS no puede ser producida simultáneamente con un idioma hablado. La organización dominante de los idiomas hablados es temporal, es decir, las palabras se suceden en el tiempo. Lo que sucede cuando uno habla una lengua y al mismo tiempo utiliza las señas, es que este tipo de organización temporal y secuencial pasa a dominar la producción. Las señas se subordinan a la lengua hablada, siguiéndose una tras otra sin las características lingüísticas que se encuentran en la simultaneidad y la utilización espacial de las LS naturales (Svartholm, 2010). De ello se deduce que la información lingüística producida por estas señas es reducida y fragmentada. Las expresiones de las relaciones gramaticales entre las señas desaparecen. También hay una gran cantidad de información importante en el discurso que no puede ser completamente expresada de un modo comprensible visualmente, como los elementos prosódicos, que incluyen la entonación y el acento (Bergman, 1977; Svartholm, 2007). Así, hacer señas y hablar al mismo tiempo podría ser utilizado para mensajes simples, cortos, pero cuando la información se vuelve más compleja, el uso de la propia lengua se vuelve una necesidad.

El reconocimiento de la relevancia de la LS en la comunicación de las personas sordas y del derecho de estas personas a ser educadas en esta lengua, lleva al desarrollo de propuestas de educación bilingüe o bilingüe-bicultural para los niños y jóvenes sordos. Estas defienden el uso de la LS en la educación del sordo y el desarrollo paralelo de la lengua correspondiente a la cultura en que el individuo se inserta, principalmente a través de la exposición a esta a través de textos escritos (Johnson, Liddell y Erting, 1989). Estas propuestas consideran la LS no solo como un medio de comunicación que puede presentar ventajas en la educación de sordos, sino como una lengua completa sobre la cual es posible sentar las bases para el aprendizaje de una segunda lengua (Hugounenq, 2005). Esto implica la puesta en práctica de dispositivos pedagógicos enfocados a la construcción de un puente que vincule la enseñanza de la LS y la lengua escrita, permitiendo el desarrollo equilibrado de cada una de ellas (Mugnier, 2006). En sus inicios, la educación bilingüe para el sordo se desarrolló con fuerza en Suecia (Svartholm, 1984), donde fue aplicada de manera sistemática y rigurosa en el sistema de educación pública. Es por esto que es relevante describir algunos antecedentes y aspectos centrales de este modelo. 


\subsection{EL MODELO SUECO DE EDUCACIÓN BILINGÜE}

La educación bilingüe para sordos en Suecia, en donde la LS es considerada la primera lengua para niños sordos y la lengua de la sociedad mayoritaria la segunda, data de 1981. Ese año, el Gobierno sueco decidió que las personas sordas tenían el derecho a ser bilingües en LS Sueca y sueco. Dos años más tarde, se introdujo el primer Currículum Nacional Bilingüe en las escuelas especiales para sordos e hipoacúsicos. En ese momento se estableció que la LS debía ser la lengua usada en loa instrucción de los alumnos sordos y que la enseñanza de esta lengua, así como del sueco -principalmente en su forma escrita- debía tener como objetivo garantizar un desarrollo hacia el bilingüismo (LGR 80, 1983). La decisión sobre el uso de la LS en lugar del "sueco signado" fue tomada, en gran medida, sobre la base de la investigación lingüística que estaba en curso en ese momento. La presión y la información de las organizaciones involucradas, tales como SDR, la Asociación Nacional de Sordos de Suecia, en colaboración con las organizaciones de padres, también fue importante en este proceso (Svartholm, 1993).

En 1994 este Currículum Nacional fue reemplazado por uno nuevo en donde las demandas de las escuelas para sordos e hipoacúsicos fueron mayores: a partir de entonces, se plantea que después de diez años de escolaridad, los alumnos deben ser bilingües. Esto significa que deben leer (i.e; entender) tanto en LS como en sueco, y también deben ser capaces de expresarse en señas y por escrito. Para la asignatura de inglés, la cual es obligatoria para los alumnos sordos al igual que para los estudiantes oyentes en Suecia, el objetivo es que puedan comunicarse por escrito. Para todas las otras materias escolares, los objetivos son explícitamente los mismos que para los niños oyentes (LpO94, 1994).

El período comprendido entre estos dos planes de estudio puede ser calificado como muy intenso, tanto en la investigación como en las actividades de desarrollo dentro de las escuelas. Dos nuevos temas académicos, la LS y 'El sueco como segunda lengua para los sordos' se estaban desarrollando en la Universidad de Estocolmo, haciendo posible para los estudiantes sordos estudiar no sólo su propia lengua, sino también el sueco escrito desde una perspectiva comparativa. Una base importante para entender los procesos específicos implicados en el aprendizaje de un idioma, fue encontrada en la investigación en relación a la interlengua (e.g., Svartholm 1984, 1987a, 1987b, 1988). Esta se refiere a estudios acerca de las reglas que se observan en las producciones escritas en sueco de los sordos que aprenden sueco como una segunda lengua, en comparación con aprendices oyentes de una segunda lengua. Muchas de las "peculiaridades" que se encuentran en la lengua escrita de los niños sordos, descritas como tales por las investigaciones tempranas, resultaron no ser peculiares en absoluto. En cambio, los "errores" y "faltas" encontrados en sus escritos pueden ser explicados de la misma forma que en los aprendices oyentes de un segundo idioma: como el resultado de las estrategias activas de procesamiento del lenguaje, tales como la simplificación, la sobregeneralización y el aprendizaje holístico de palabras y frases. Es en esta perspectiva que debe verse el uso erróneo, por ejemplo, de las preposiciones y las inflexiones, así como el uso desviado, no estándar del vocabulario (Svartholm, 2008).

A partir del análisis comparativo de los dos idiomas, así como de la observación del desarrollo de los niños dentro de las escuelas, los investigadores y profesores fueron aprendiendo gradualmente más sobre la lengua escrita desde la perspectiva del niño sordo. En el modelo bilingüe, desde el principio el profesor hace hincapié en que la LS y la 
lengua escrita son dos lenguas diferentes con estructuras distintas. Puesto que no hay correspondencia uno-a-uno entre las palabras y las señas, no hay uso de la traducción de textos escritos en señas palabra por palabra. En cambio, el significado de frases completas y párrafos debe centrarse en la traducción y en explicaciones posteriores en relación con el vocabulario y la gramática. Trabajar con textos, "reales" escritos para niños, que no sean para uso pedagógico, sino para el entretenimiento o para la presentación de hechos u otros fines auténticos, es fundamental. Los niños sordos necesitan, al igual que cualquier estudiante de idiomas, un input lingüística rica y variada y la necesidad de esto difícilmente se puede cubrir con textos cortos y simplificados, especialmente adaptados. Este input se hace inteligible para el niño a través de la LS, en las explicaciones y traducciones presentadas por el profesor (Svartholm, 2008).

Por otra parte, dado que el niño sordo no tiene o solo tiene escasas habilidades en la lengua oral, como para apoyarse en estas para el aprendizaje de la lectura, los procesos de aprendizaje de la lectura y el aprendizaje de la lengua no pueden separarse el uno del otro; el niño construye conocimiento acerca de la lengua sobre todo a través de la lectura.

Después de más de 25 años en los que se ha desarrollado el modelo bilingüe para la educación de sordos en Suecia, los resultados han sido muy positivos. Cuando los escolares sordos se gradúan -un año después que los estudiantes oyentes- rinden el mismo test nacional en sueco, al igual que estos últimos. Entre 2002 y 2008, en promedio, los estudiantes de las escuelas de sordos aprobaban con un nivel de desempeño del 59\%, lo que demuestra un nivel de lectura que les permitiría aprobar igualmente la escuela regular (SPM 2002-2007, SPSM 2008). Es importante señalar que se incluye aquí a estudiantes que tienen problemas específicos de aprendizaje o presentan una situación lingüística compleja en su hogar por pertenecer a familias de inmigrantes, lo cual es cada vez más común en las escuelas de sordos en Suecia (Hendar, 2008). Un grupo creciente también son los niños que han comenzado sus estudios en una escuela regular y luego se han cambiado a una escuela especial debido a problemas de lenguaje. Claramente, no se puede esperar que estos niños desarrollen el bilingüismo en forma tan eficiente ya que, por lo general, carecen de conocimientos de la LS antes de ingresar a la escuela especial (SPM, 2007).

¿Qué pasa entonces con los resultados de otros enfoques bilingües en la educación de sordos? Alrededor de 1990 hubo un movimiento en el campo de la educación de sordos en EE.UU. y Canadá hacia la adopción de lo que fue llamado el modelo bilingüebicultural $(\mathrm{Bi} / \mathrm{Bi})$ para la enseñanza del idioma y la alfabetización de los estudiantes sordos (Gibson et al., 1997; Mashie, 1995; Strong, 1995). Sin embargo, este concepto no fue ampliamente difundido. Solo un $9 \%$ de los 45.000 estudiantes sordos en EE.UU. durante el año 1999 asistió a una escuela o clase que decía trabajar en forma bilingüe, la mayoría de ellas, sin embargo, sin un currículum formal (LaSasso, 2000; LaSasso y Lollis, 2003). No hay mucha información disponible sobre los resultados de estas experiencias. La educación de sordos en EE.UU. todavía presenta un enfoque basado principalmente en la Comunicación Total/inglés signado, relevando también la comunicación oral. No obstante, el Lenguaje de Señas Americano (ASL) tiene una fuerte posición dentro de la comunidad sorda. En un contexto internacional más amplio, es aún más difícil encontrar resultados que puedan ser comparados con los reportados en las escuelas suecas, ya que: “...todavía es relativamente poco común para los estudiantes sordos y con problemas de audición de las escuelas especiales, seguir el currículum escolar gubernamental de sus 
respectivos países. En este sentido, nosotros en Suecia planteamos altas exigencias a nuestros alumnos sordos e hipoacúsicos" (SPM, 2005: 9).

\subsection{EDUCACIÓN DE SORDOS EN CHILE}

Al igual que en la mayoría de los estudios internacionales, los resultados de la educación de sordos en Chile, específicamente en lo que se refiere a la lectura y la escritura, están lejos de ser satisfactorios, encontrándose que los alumnos sordos muestran un desempeño muy por debajo de lo esperado para su edad (Lissi et al., 2003; Miranda, 1997).

La educación formal para sordos en Chile data de 1852, año en que fue fundada la primera escuela para niños sordos en el país, la que hoy es llamada Escuela de Niños Sordos Anne Sullivan (Herrera, 2010; Oviedo, 2008). Actualmente, en los establecimientos educacionales existe una diversidad de enfoques pedagógicos, que corresponden a diferentes ideologías sobre la educación y las personas sordas: oralismo, comunicación total, auditivo oral, bicultural-bilingüe. El fuerte arraigo del oralismo, junto con su ideología sobre las personas sordas y su educación, ha puesto mucha resistencia a los cambios (Adamo, Cabera y Acuña, 2008).

A partir de la década de los 90, un equipo de investigadoras en la Universidad Metropolitana de Ciencias de la Educación desarrolla una serie de estudios lingüísticos sobre la Lengua de Señas Chilena (LSCh) (Acuña et al., 1999; Adamo et al., 1999, Adamo et al., 2003). Estas mismas autoras paralelamente comienzan a plantear la necesidad de una educación bilingüe para los estudiantes sordos en Chile (Adamo et al., 1997; Adamo, Cabrera y Acuña, 2008).

Hacia el año 1998 en la escuela de niños sordos “Dr. Jorge Otte Gabler”, se empieza a gestar un modelo de educación bilingüe. En el año 2000 se da inicio en esta escuela al Proyecto Educativo de Educación Bilingüe-Bicultural, aprobado por el Ministerio de Educación. Esta es la primera escuela en el país en adoptar este modelo educativo y actualmente cuenta con la participación de personas sordas, quienes se desempeñan como modelos de la cultura sorda y modelos lingüísticos de la LSCh. Los adultos sordos participan del proceso educativo al interior de la sala de clases, como profesores, co-educadores o instructores de LS. Por su parte, los profesores y profesoras oyentes imparten educación a través de la LS. El programa contempla la enseñanza del español como una segunda lengua, dando primordial importancia a la enseñanza de la lengua escrita (Adamo, Cabrera y Acuña, 2008).

A pesar de que ha habido avances en la incorporación de la LS a las escuelas, se está muy lejos de la adopción de un modelo de educación bilingüe a nivel nacional, aunque la nueva Ley 20.422 (2010) reconoce a la LS "como medio de comunicación natural de la comunidad sorda" (Artículo 26).

\section{PRÁCTICAS Y ESTRATEGIAS UTILIZADAS EN LA ENSEÑANZA DE LA LENGUA ESCRITA A LOS SORDOS EN CONTEXTOS DE EDUCACIÓN BILINGÜE}

Los antecedentes presentados hasta aquí dan cuenta de los principios y lineamientos que se consideran centrales para un modelo bilingüe, como el que se ha estado 
implementando en Suecia desde hace ya más de 25 años. Como ya ha sido mencionado más arriba, en el contexto chileno las experiencias de educación bilingüe para el sordo tienen una trayectoria de origen más reciente. Por otra parte, las políticas educativas a nivel nacional y el marco legal no son aún comparables con la situación descrita en Suecia.

En esta sección se describen algunas prácticas y estrategias específicas, que caracterizan la forma en que se trabaja en contextos de educación bilingüe para estudiantes sordos, con un énfasis en la forma en que la LS es utilizada en el proceso de enseñanza-aprendizaje de la lengua escrita en estos contextos. ${ }^{1}$ Para hacer más cercana esta presentación, se ha optado por apoyarse en ejemplos tomados de un contexto chileno, que permiten ilustrar algunos aspectos importantes del trabajo educativo en establecimientos de educación que adoptan un modelo bilingüe para la enseñanza de los alumnos sordos. Los ejemplos específicos que se incluyen fueron seleccionados de un amplio material audio visual que fue recolectado como parte de un proyecto de cuatro años de duración, ${ }^{2}$ en el que se siguió a tres grupos de niños y sus profesoras en una escuela con un proyecto educativo bilingüe/bicultural, con el objetivo de describir y analizar las estrategias utilizadas por las profesoras y evaluar el desarrollo de las competencias en LS y lengua escrita por parte de los alumnos.

En primer lugar, se presentan algunos aspectos relevantes de la estructura general de la clase en el aula bilingüe, para luego analizar algunos ejemplos del trabajo específico con material escrito. ${ }^{3}$

\subsection{CONSIDERACIONES GENERALES RESPECTO A LA ORGANIZACIÓN DE LA CLASE}

Dentro del contexto de educación bilingüe para sordos, es importante considerar no solo las estrategias específicas de enseñanza aprendizaje desplegadas por los docentes, sino también aspectos tanto de la organización de la clase como de las condiciones y el ambiente en que ésta se desarrolla.

La estructura general de la clase está organizada de manera tal que permite brindar a los niños un marco de trabajo y un contexto previo a los temas y contenidos que se abordarán. En un inicio, los profesores conversan con los niños acerca de los temas que han visto recientemente o recuerdan alguna actividad significativa que se relaciona con la clase que realizarán. Luego, hacen preguntas abiertas para ver qué y cuánto recuerdan los alumnos e introducen el tema señalando lo que harán en esa clase. Un ámbito relevante a considerar es el mantenimiento de la atención y el acceso a la información de parte de los alumnos. La distribución espacial de los alumnos dentro del aula, así como el formato de los materiales y recursos de apoyo que se utilizan durante las clases, permite que todos los niños puedan acceder visualmente a la información que se está entregando y que la comunicación entre ellos no sea obstaculizada. Los niños se sientan en círculo

\footnotetext{
Muchas de las prácticas y estrategias que se detallan aquí se basan en el trabajo desarrollado por K. Svartholm, una de las autoras de este artículo, investigando y apoyando la labor de los profesores de sordos en las escuelas suecas durante los últimos 25 años.

2 Proyecto Fondecyt 1060762: "LS y lengua escrita en niños sordos. Su adquisición y posibles interacciones en un contexto educativo bilingüe".

3 Dado que en esta sección se hablará de la forma en que se trabaja en el enfoque bilingüe de manera general, ilustrando algunos aspectos a través de ejemplos de actividades desarrolladas por profesoras chilenas, se usará "profesores" en términos genéricos y "profesoras" cuando se aluda a los ejemplos específicos.
} 
o semicírculo con la mirada orientada hacia la profesora, a la vez que pueden observar alternativamente a sus compañeros. Se buscan formas de facilitar el trabajo colectivo en la lectura y escritura, por ejemplo, el proyectar en la pizarra o en un telón los textos escritos y el material visual de apoyo.

Por otra parte, los profesores realizan diversas acciones para que los alumnos sigan visualmente aquello que está ocurriendo frente a ellos y tengan pleno acceso a lo que dicen sus compañeros. Por ejemplo, indicando a los niños que se desplacen al frente de la sala para que los demás puedan verlos, así como dirigiendo la atención del resto hacia aquel niño que está comunicando algo.

Un aspecto relevante tiene que ver con las acciones que los profesores pueden llevar a cabo para asegurar que los alumnos puedan seguir en todo momento la clase y no pierdan el hilo de esta durante el proceso. Es extremadamente importante que el profesor mantenga informados a los niños respecto a la estructura de la clase y el desarrollo de ésta, ya que el niño sordo es visualmente dependiente, por lo que si mira hacia otro lado durante un par de segundos el profesor podría cambiar el tema y el niño podría perderse. Para esto, pueden emplearse diversos apoyos tales como escribir la estructura de la clase en la pizarra, indicar la secuencia de temas a tratar y asignarles números para hacer referencia a estos durante la clase. Otra forma de ayudar al niño a no perder el hilo de la clase es apoyarse en estrategias que utilizan las personas sordas para comunicarse en LS. Por ejemplo, una forma de ubicar al otro cuando se está hablando de más de un tema es usar los dedos de una mano y si por ejemplo, hay cuatro temas, puedo hacer referencia al primer punto, señalando a mi dedo índice y así sucesivamente usando los demás dedos, volviendo a señalar el dedo correspondiente cuando hago referencia a ese tema. Esta estrategia se ha encontrado en La LS americana, sueca y noruega (Liddell et al., 2007). Otro ejemplo es utilizar la seña "terminar" cuando se finaliza un tema, de manera que se muestre claramente que se pasa a otro tema.

\subsection{EL TRABAJO CON MATERIAL DE LECTURA}

Las distintas estrategias que despliegan los profesores al momento de trabajar con material de lectura cumplen diversas funciones, tales como: explicitar a los niños los elementos que hay que considerar al momento de leer en LS, asegurar la comprensión durante la lectura y aprovechar la actividad de lectura para enseñar algún aspecto lexical o gramatical de la lengua escrita.

Las actividades de lectura involucran el trabajo con distintos tipos textos, los que pueden ser leídos de forma individual, en pequeños grupos de dos o tres alumnos o de manera colectiva en conjunto con el profesor.

a) Forma de leer cuando se utiliza la LS. Cuando se utiliza la LS en la lectura de textos, los profesores enfatizan las diferencias entre la LS y la lengua escrita, destacando la importancia de respetar los elementos y la sintaxis de la LS al leer para mantener la fluidez en la expresión. Además, se aprovecha esta instancia para enseñar aspectos específicos de las dos lenguas. A continuación se presentan algunos ejemplos:

- La profesora se prepara a leer junto a los niños un texto proyectado en la pizarra, se trata de un ejemplo de una descripción, específicamente la descripción de una abuela. El texto dice: La abuela es alta y delgada. Ella tiene la piel clara, el pelo 
canoso, la piel arrugada y los ojos claros. Ella es simpática y está alegre. Antes de comenzar a leer la profesora dice: "acuérdense lo que uno hace cuando usa LS para leer, primero leo un trozo (indica un trozo del texto con sus dedos) y luego seño, luego leo otro trozo (señala otra vez el texto con el dedo) y luego seño". La profesora lee la primera oración del texto: "l-a, a-b-u-e-l-a, ES, ALTA, Y..." y luego les dice "no, en señas primero leemos todo el párrafo, ¿se acuerdan? Primero yo miro el texto, entiendo lo que dice (simula leer el texto, recorriéndolo con su dedo), lo retengo, lo cambio y lo digo en señas ¿Entienden?"

- Durante la lectura colectiva de un cuento, un niño lee frente a sus compañeros la oración jugar a la pelota. Al hacerlo seña "JUGAR - a (deletrea a)". La profesora detiene al niño que estaba leyendo y dirigiéndose a todos dice: "recuerden lo que les expliqué antes, ¿Se dice: JUGAR a-1-a. PELOTA? ¿Se dice así?”. Los niños responden "no". Ella dice "no" y les pregunta “¿cómo?" Otro niño dice "JUGAR PELOTA... nada más". Más adelante, otro niño está leyendo la oración: lo que más le gusta es jugar. El niño utiliza la seña "ES". La profesora explica: "Cuando escribimos en castellano, para que esté bien escrito debemos poner es, pero en LS no se usa "ES". - Durante la lectura grupal de un cuento breve, los niños se encuentran con la expresión todos los días en castellano escrito. La profesora utiliza esta oportunidad para mostrar que para expresar esto en LSCh no se puede indicar cada palabra de la oración si no que se debe utilizar una seña específica que significa "todos los días".

En estos ejemplos, las profesoras enfatizan que cuando se lee un texto utilizando la LS, no se debe realizar una lectura "palabra-por-palabra", ya que eso conlleva una alteración de la manera en que se expresan ideas en esta lengua. Así también, para que la lectura en señas sea fluida, es necesario considerar unidades de sentido completas, como las oraciones, para comprender primero el significado del texto y luego expresarlo en LS. En los ejemplos también se observan referencias a la comparación entre la LS y la lengua escrita, específicamente al mostrar que algunos elementos gramaticales que son propios del castellano no se utilizan al momento de leer en LS y que la forma de expresar una misma idea puede ser distinta dependiendo cuál de estas lenguas se use.

b) Asegurar la comprensión durante la lectura. Para asegurar la comprensión, los profesores implementan estrategias tales como: dar a conocer el tema general del texto que van a leer, explicar el significado de algunas palabras que aparecen en el texto o corregir cuando los niños confunden una palabra con otra. En estas actividades, también se ve reflejado el trabajo del vocabulario en contexto y el aprovechamiento de las oportunidades que entregan los textos para mostrar aspectos relevantes del castellano.

Durante la lectura de un texto, los niños sordos se enfrentan con muchas palabras que no conocen y cometen errores, atribuyéndole un significado incorrecto a una palabra. En el siguiente ejemplo, al darse cuenta la profesora que la seña que están produciendo los

4 Al transcribir intervenciones de la profesora y/o los alumnos se ha usado el siguiente formato: minúscula entre comillas indica un texto originalmente en LS traducido al castellano; mayúscula entre comillas cuando se registra en castellano un ejemplo de forma correcta o incorrecta de expresar algo en LSCh; letras separadas por guiones cuando se deletrea manualmente algo; cursiva refiere a algo que está escrito; texto entre paréntesis describe una acción. 
niños no corresponde a la palabra que está en el texto, interviene, mostrando la diferencia ortográfica que existe entre ambas palabras, para evitar errores en la comprensión.

- Durante la lectura colectiva de un texto, los niños al ver escrita la palabra cosa, leen "CASA". La profesora explica en LS el significado de ambas palabras; primero las escribe en la pizarra, y luego muestra la letra que las distingue $(\mathrm{a}-\mathrm{O})$ y las marca para que los niños observen la diferencia entre ambas. Más adelante, hace lo mismo con las palabras hoy y hay y las palabras pero y para.

En el ejemplo que sigue, se observa cómo, frente a una palabra que los niños no conocen, la profesora aprovecha de explicar su significado a partir de los conocimientos que ellos tienen. En este caso, la profesora recurre a un morfema que es familiar para ellos (pan).

- Durante la lectura colectiva de un texto, la profesora explica el significado de la palabra panadera. Primero, escribe la palabra panadera en la pizarra, encierra en un círculo las 3 primeras letras pan, los niños reconocen la palabra y hacen la seña correspondiente. La profesora los refuerza y luego, apuntando al resto de la palabra y al dibujo donde aparece una panadera, explica que la palabra completa significa "una persona, que es mujer, que vende y hace pan".

En otra ocasión, la aparición de cierta palabra en un texto es utilizada como oportunidad para mostrar que ésta tiene más de un significado, dependiendo del contexto en que se utilice.

- La profesora presenta un texto escrito por ella en el que recuerda y describe a su abuela. Al llegar a la oración Ella me cocinaba ricos queques y dulces, marca la palabra rico en el texto y la escribe en la pizarra. Luego, apunta a la palabra, la deletrea y dice "Esta palabra puede significar "RICO", que tiene mucho dinero o también puede significar "SABROSO".

\subsection{EL TRABAJO EN LA PRODUCCIÓN DE TEXTOS ESCRITOS}

El trabajo en escritura involucra la producción de textos en forma individual o en parejas y actividades de trabajo con el grupo completo. Una forma en que se combina la escritura individual con el trabajo grupal en producción escrita, es la corrección colectiva de los textos creados por los niños. En estas ocasiones, luego de que los niños han generado su versión preliminar del texto, la profesora escoge uno de ellos, lo copia en la pizarra y pide al autor que pase al frente a leerlo en LSCh, luego se realiza una corrección grupal de lo que éste escribió, produciendo una nueva versión que también se va escribiendo en la pizarra. Esta es una oportunidad para que las profesoras enfaticen algunos aspectos específicos de la escritura en castellano.

La utilización fluida de la LS en el aula, posibilita el desarrollo de actividades que llevan a generar textos de mejor calidad. Una conversación fluida en LS previa a la escritura, posibilita una producción más rica ya que permite discutir acerca del tema y 
generar una mayor diversidad de ideas. De forma inversa, una vez que el niño produce un texto, puede explicar en LSCh lo que quiso escribir, lo que facilita el andamiaje por parte de la profesora, de manera que la escritura refleje de la forma más fidedigna sus ideas. A continuación presentamos un ejemplo que ilustra algunos de estos aspectos:

- La profesora realiza un proyecto con los niños en el que cada uno escribirá sobre su historia escolar. La idea es que los alumnos narren primero en LSCh lo que van a escribir y luego produzcan un texto escrito. El día en que los niños narran sus historias en LS, se realiza una filmación de cada relato. Luego se proyectan los videos y recién ahí comienzan a producir los textos escritos. Esta producción se realiza de manera individual, con algún apoyo de la profesora cuando los niños lo solicitan. El desarrollo de la actividad dura varias semanas, ya que se dedica una sesión de clase para trabajar corrigiendo en conjunto uno de los textos producidos por los niños. En estas sesiones, la profesora copia el texto de un niño en la pizarra y en conjunto con el curso, va revisando el texto oración por oración. En cada una de estas sesiones se proyecta primero la narración en LS antes de comenzar a corregir el texto escrito.

Este ejemplo muestra también cómo la filmación de los niños narrando lo que desean contar en LSCh, permite llevar un registro de lo que el niño quiso decir originalmente, y así poder volver sobre esta idea una vez que se comenzó a escribir.

Como ya hemos señalado anteriormente, dado que en el aula bilingüe estamos trabajando con dos lenguas diferentes, es necesario enfatizar que al escribir un texto que fue producido previamente en LSCh, lo que estamos realizando es una traducción de una lengua a otra. El trabajo en producción de textos es una muy buena instancia para realizar comparaciones entre las lenguas, de manera de ir desarrollando una mayor conciencia metalingüística, y también una oportunidad para enfatizar aspectos específicos de la gramática del castellano, en contextos significativos. El siguiente ejemplo ilustra lo anterior.

- La profesora indica a los niños que escriban, de manera individual, un texto que responda a la siguiente pregunta: “Cómo celebraste el día del sordo?” Para elaborar los textos, los niños tienen como apoyo las fotos de la celebración que se hizo en el colegio. Luego que cada niño termina la tarea, el curso junto a la profesora edita uno de los textos de manera colectiva. En este proceso, la profesora enfatiza que cuando escribimos en castellano cambiamos la forma de expresar una idea, en relación a cómo lo decimos en LS. Por ejemplo, a partir de la revisión de un texto producido por uno de los alumnos, que en una parte decía yo contento, ella escribe en la pizarra L1: Yo contento. Luego seña "YO CONTENTO" y agrega: "pero cuando escribo, debo utilizar L2", y escribe en la pizarra L2: yo estoy contento. Luego explica que lo que le faltaba al texto escrito por el niño es el verbo estar, que debe incluirse al expresarse en castellano.

\subsection{ACTIVIDADES ESPECÍFICAS EN LAS QUE SE REALIZAN COMPARACIONES ENTRE LAS LENGUAS.}

Como ya se vio más arriba, durante las actividades de lectura y escritura las profesoras aprovechan distintas situaciones para mostrar diferencias entre el castellano y la 
LSCh. En otras oportunidades, este tipo de explicaciones se realizan en el contexto de una actividad dedicada justamente a mostrar este tipo de contrastes, tal como se ilustra en los ejemplos siguientes.

- En un primer momento, la profesora muestra 3 oraciones escritas en la pizarra e indica como en cada una de ellas se usa la palabra perdió, pero la seña que se usa al traducirlas a LSCh es diferente en cada caso:

1. El 3ro Básico B perdió $2-5$.

2. A Javiera se le perdió el cuaderno.

3. Mi mama se perdió las noticias

En otro momento de la clase presenta las siguientes oraciones, mostrando como cada una de ellas usa una expresión distinta en castellano, pero al traducirlas a LSCh se observa que una misma seña es utilizada en los tres casos.

1. Mi mamá no tiene $\$ 10.000$.

2. Francisca no vino hoy.

3. Mi lápiz no estaba en la mochila.

\subsection{COLABORACIÓN ENTRE PARES}

La colaboración entre pares puede darse tanto de manera espontánea, como a solicitud de la profesora durante el trabajo en clases. Una comunicación fluida entre los alumnos, posibilitada por la LS, otorga mayores oportunidades de realizar un trabajo colaborativo. Dado que los grupos son heterogéneos tanto en el manejo de la LS, como en el manejo del castellano escrito, los estudiantes pueden apoyarse entre ellos para lograr una mejor comprensión de las lecturas y una mayor riqueza en la producción de textos escritos. En los ejemplos que siguen se observan actividades de trabajo colaborativo propuestas por las profesoras.

- Luego de haber leído en parejas un cuento simple y haberlo compartido con sus compañeros, las mismas parejas desarrollan por escrito una guía de trabajo acerca del cuento. Durante el transcurso de la actividad, se observan distintas formas en que los niños colaboran entre sí: leyéndole al compañero lo que dice en alguna parte del cuento, indicándole en el texto la palabra que debe utilizar, deletreándole la palabra que quiere que escriba, recordándole ciertos aspectos formales de la escritura, entre otras.

- Luego de haber visitado el Zoológico Parque Metropolitano, la profesora desarrolla una actividad de producción colaborativa de textos en grupos de 3 o 4 niños, en la que ellos deben contar a sus padres qué hicieron ese día. Para esto parte haciendo un recordatorio de las actividades que hicieron en el zoológico, apoyándose en algunas fotos disponibles en la web. Los niños comentan qué animales vieron, qué comieron y cómo fue el recorrido. Al inicio del trabajo en grupo, los niños designan a un compañero para que escriba y los demás van comentando y señalándole qué debe escribir. La profesora los incita a que cuando no sepan cómo escribir una palabra, les pregunten a un compañero, y se apoyen entre ellos utilizando los recursos de que disponen dentro de la sala (e.g., cuadernos, papelógrafos). 


\section{DISCUSIÓN}

En las páginas anteriores hemos expuesto algunos antecedentes respecto a los fundamentos de un modelo bilingüe para la educación de niños sordos, con un foco especial en el modelo sueco, dado que este país fue pionero en implementar de manera sistemática un enfoque con estas características. Hemos incluido también en la descripción de algunas de las prácticas asociadas a este enfoque, centrándonos en la enseñanza de la lengua escrita. Para esto hemos recurrido a ejemplos que ilustran algunos de los aspectos centrales de este proceso.

En esta última sección queremos reflexionar acerca de lo anteriormente expuesto, considerando la importancia de avanzar en la línea de implementar más programas con estas características en nuestro país y fortalecer aquellos que ya están en funcionamiento. Para esto queremos plantear una discusión en torno a tres preguntas: (a) ¿Cuáles son las ventajas que ofrece el modelo bilingüe, y particularmente el uso de la LS en el aula, para el proceso de enseñanza-aprendizaje del castellano escrito? (b) ¿Qué aspectos son centrales en el trabajo de aula cuando nos situamos en este enfoque? y (c) ¿Cómo podemos continuar profundizando en el desarrollo de contextos educativos bilingües de calidad en Chile y en otros países en los que no está aún consolidado?

\subsection{OPORTUNIDADES QUE OFRECE LA LS PARA EL PROCESO DE APRENDIZAJE}

A partir de lo descrito anteriormente, es claro que la educación bilingüe en general, y específicamente, el uso de la LS en la sala de clases, presenta una serie de ventajas y oportunidades en el proceso de enseñanza del castellano como segunda lengua para el sordo, específicamente en su modalidad escrita. En términos generales, es evidente que la falta de una lengua bien desarrollada dificulta el acceso a los contenidos escolares y la participación en el proceso de aprendizaje (Svartholm, 2010). La educación bilingüe para el sordo, con su énfasis en la adquisición temprana de la LS y la utilización de esta en el aula por parte de los profesores, otorga entonces mayores oportunidades de tener una experiencia educativa plena, sin las restricciones que impone el limitado desarrollo de la lengua oral por parte de los estudiantes.

Por otra parte, la LS ofrece muchas ventajas, que permiten aproximarse a la enseñanza de la lengua escrita de una manera más efectiva, lo que se refleja en los resultados positivos obtenidos por los estudiantes suecos que han sido educados en el modelo bilingüe ((SPM 2002-2007, SPSM 2008). A continuación se resumen algunas de estas ventajas.

- Fluidez en la comunicación profesor-alumno. La comunicación fluida en LS entre el profesor y sus alumnos posibilita una serie de prácticas, actividades y procesos de una manera mucho más eficiente y efectiva que lo que se observa en los contextos monolingües, donde la única lengua utilizada es el castellano, en su forma oral y escrita, con las restricciones que esto impone a la comunicación para el sordo. Lo anterior se ve reflejado, entre otras cosas, en la posibilidad de trabajar con textos complejos, cuyo contenido y formato son acordes a la edad e intereses de los alumnos, más que a su conocimiento de la segunda lengua. Esto porque la comunicación fluida entre el profesor y alumnos en LS permite traducir partes del texto del castellanos a la LS, explicar conceptos que puedan ser difíciles de comprender por los 
alumnos, y monitorear más directamente la comprensión de los textos leídos. En el caso de las actividades de escritura, la comunicación en LS facilita el trabajo de producción de textos, al poder conversar de manera más extensa y rica respecto a lo que el alumno quiere comunicar. Esta fluidez en la comunicación profesor alumno posibilita también la realización de actividades previas y posteriores al desarrollo de las tareas de lectura formal o producción escrita de textos (Schirmer, 2000) en las que los niños tengan una participación activa. Por otra parte, el manejo de una primera lengua permite potenciar el desarrollo de procesos metacognitivos que, tal como lo plantean Palincsar y Brown (1984), resultan relevantes en la adquisición y despliegue de estrategias de comprensión lectora, ya que el profesor puede modelar y proveer de un andamiaje adecuado en este proceso.

- Fluidez en la comunicación entre los alumnos. La adquisición de la LS como primera lengua permite a los alumnos sordos comunicarse más efectivamente entre ellos, posibilitando que en la sala de clases se pueda dar una conversación colectiva entre el profesor y los alumnos, y no solo un diálogo uno a uno entre cada alumno y el profesor. Esta fluidez en la comunicación permite también la realización de actividades de trabajo colaborativo, las que a su vez potencian no solo el aprendizaje de los contenidos, sino también el desarrollo lingüístico en la L1 y la L2, dado que los alumnos presentan diferentes niveles de desempeño en una y otra lengua.

\section{- Posibilidad de utilizar el conocimiento de una lengua para enseñar y aprender} una segunda lengua. Los enfoques monolingües en la educación de sordos, en los que el idioma hablado del país es la forma prioritaria de comunicación y aquella en que se imparten todos los contenidos escolares, plantean varias limitaciones en el proceso de enseñanza para estos alumnos. En la mayor parte de los casos, el niño ingresa al sistema escolar sin una lengua bien desarrollada, por lo que debe desarrollar esta lengua al mismo tiempo en que se espera que aprenda los demás contenidos exigidos por el currículum. Por otra parte, la enseñanza de la lengua mayoritaria del país, en nuestro caso el castellano, se ve limitada por las restricciones que la sordera impone al input en modalidad oral (Marchesi, 1987, 2001) y las dificultades derivadas de aprender una lengua a partir solo de su modalidad escrita, en ausencia de una primera lengua. Como se ha mostrado en los ejemplos descritos en la sección anterior, las posibilidades que ofrece la LS de traducir de una lengua a otra, explicar significado de vocabulario y expresiones nuevas para el niño en su primera lengua, y la comparación entre ellas, permiten un mejor acceso a la segunda lengua.

\subsection{CONSIDERACIONES GENERALES PARA EL TRABAJO EN CONTEXTOS BILINGÜES}

- Atención visual. En contextos donde la comunicación se realiza a través de la LS, es fundamental lograr mantener la atención visual de los niños para que no solo puedan acceder a los contenidos, instrucciones y preguntas presentados por la profesora, sino también lograr una comunicación de calidad entre ellos. Las actividades específicas antes descritas, en las que se favorece el trabajo grupal con todo el curso o en pequeños grupos, relevan la importancia de este aspecto en el aula. Como se mencionó anteriormente, para cumplir este requisito hay condiciones físicas de la sala de clases, en términos de 
luminosidad y disposición de los puestos de los niños en el aula, entre otras, que deben tenerse presentes para favorecer una buena percepción visual que permita pleno acceso a lo que los demás están comunicando en todo momento. Además, es importante enseñar a los niños a respetar los turnos para comunicarse, a establecer contacto visual cuando se le da la palabra a alguien, a esperar el momento de hablar hasta que todos puedan verse entre sí (Erting 1988; Mather 1987; Svartholm, Andersson y Lindahl, 1993).

- Proceso de enseñanza-aprendizaje de la L1 y la L2. Si estamos planteando la necesidad de que en la escuela de sordos tengan clara presencia, tanto la LS como el castellano en su modalidad escrita, no solo se trata de que el conocimiento de la L1 facilite el aprendizaje de la L2, sino que debe haber espacio para que ambas lenguas se desarrollen durante los años escolares (Mugnier, 2006). Adquiere así importancia la enseñanza de la LS como una materia escolar. Por otra parte, es importante desde el comienzo que los niños comprendan que el castellano y la LS son dos lenguas distintas y que por tanto no hay una correspondencia uno-a-uno entre los elementos de una y otra. Para esto, es fundamental separar una lengua de otra en el proceso de enseñanza (Hermans et al., 2008; Svartholm, 1993). A su vez, la comparación entre las lenguas es una parte importante de la enseñanza en un contexto bilingüe. De esta manera, el conocimiento que los niños van adquiriendo de la LS permite explicar aspectos de la segunda lengua que se enseña en la escuela. Sin embargo, tal como plantean Humphries y Allen (2008), para que esto pueda ocurrir de manera sistemática y efectiva, se requiere que el profesor de estudiantes sordos -sea sordo u oyente- tenga un buen dominio y conocimiento de ambas lenguas.

Como se vio en los ejemplos descritos anteriormente, estos dos aspectos: la separación de las lenguas y la utilización de las comparaciones entre ambas, se pueden intencionar claramente en el contexto de actividades de lectura y escritura, pero también se requiere de instancias específicas de enseñanza orientadas a este propósito.

- Colaboración. Dada la importancia de potenciar los aprendizajes y el desarrollo de la L1 y la L2 en la escuela, la posibilidad de trabajar colaborativamente en el aula adquiere doble importancia: como oportunidad para estimular el desarrollo de la lengua escrita y como instancia comunicativa que potencia el desarrollo de la LS. En la medida en que se propongan actividades en que los niños puedan trabajar juntos, en parejas o pequeños grupos para realizar tareas de lectura y escritura, se generan oportunidades en las que unos aprenden de otros. Normalmente en un mismo curso existe bastante variabilidad en el nivel de competencia lingüística de los alumnos, tanto en LS como en el manejo de la lengua escrita, lo cual permite que en la interacción se genere una zona de desarrollo próximo, que posibilite el aprendizaje y el desarrollo de todos (Vygotski, 1962).

\subsection{ALGUNAS CARACTERÍSTICAS PROPIAS DE LAS ACTIVIDADES} DE LECTURA Y ESCRITURA EN CONTEXTOS BILINGÜES

\subsubsection{Lectura}

Considerando que, como se planteó en la primera sección, el texto escrito es la vía principal de input lingüístico en la segunda lengua para los niños sordos (Svartholm, 
1984), es de gran relevancia exponer a estos estudiantes a gran cantidad y variedad de textos de calidad.

Como se ha visto hasta aquí, una de las ventajas que ofrece la educación bilingüe es la posibilidad de una buena mediación del profesor, para acercar a los niños a textos complejos que aún no son capaces de leer en forma autónoma. La fluidez en la comunicación profesor alumno a través de la LS posibilita un mejor andamiaje en las situaciones en que el niño lee un texto cuyo nivel de dificultad limitaría la comprensión que pudiera alcanzar por sí mismo. Esto permite que se pueda trabajar con textos auténticos, escritos para niños de su edad y que, por tanto, resulten motivantes para los alumnos. Por el contrario, cuando la comunicación se ve limitada por apoyarse solamente en la lengua oral, se suele presentar a los niños textos adaptados por el profesor o que han sido escritos para niños de menor edad, disminuyéndose en el primer caso, la calidad de los textos y en el segundo su potencial de resultar atractivos y de interés para los niños.

La lectura, así como las actividades de producción de textos, ofrecen oportunidades para trabajar aspectos gramaticales en contextos significativos. Por ejemplo, en el enfoque de la pedagogía de género (Gibbons, 2002), basado en la gramática funcional de Halliday (1985), se presentan a los niños textos auténticos de variado género (e.g., fábulas, descripciones, textos informativos, narraciones, etc.) y se trabajan aspectos gramaticales característicos de distintos formatos de textos escritos, en contexto.

En las actividades de lectura compartida, tal como se vio en los ejemplos presentados, se debe enfatizar la comprensión, y la posibilidad de obtener significado del contexto al enfrentar palabras nuevas, todo lo cual se ve dificultado si se realiza una lectura palabra por palabra (Johnson et al., 1989). Por otra parte, debido a que el vocabulario de los niños sordos en la L2 es limitado, ocurre muy seguido que se encuentren con palabras que no logran identificar (Lissi et al., 2010). Es importante, por tanto, enseñar a los niños que ellos no deberían detenerse cuando no entiendan, sino continuar leyendo y tratar de obtener el significado a partir del contexto. Esto se ve favorecido por el uso de estrategias metalingüísticas, las que implican verbalizar lo que está sucediendo cuando están tratando de construir significado a partir de un texto o deducir el significado específico de una palabra (Svartholm, 1998). La comprensión de los textos se comprueba fácilmente gracias al uso de la LS. Cuando los niños traducen lo escrito a LS se puede ver si están o no entendiendo, de manera que el profesor puede rápidamente aclarar confusiones y continuar con la lectura, o utilizar los errores como oportunidad para desarrollar la capacidad de cuestionarse si el significado que el niño está otorgando a la palabra encaja con el contexto o no.

\subsubsection{Escritura}

La actividad de producción de textos escritos se ve enriquecida cuando existe la oportunidad de que el profesor y los alumnos conversen acerca de lo que posteriormente van a escribir. La LS se utiliza así para hablar antes de escribir, compartir ideas y crear estímulos para el texto. A partir de una comunicación fluida en LS, el profesor puede saber con detalle qué es lo que los niños desean escribir. Esto ayuda a entender y corregir el texto producido por el alumno, de manera que lo que el profesor sugiere refleje lo que los niños realmente quieren decir. Otra ventaja de la LS para el trabajo en escritura es que puede ser usada para hablar acerca de los diferentes propósitos de esta, los diversos 
tipos de textos, y la forma en que éstos deben adaptarse para que distintas personas puedan leerlos. La presencia de dos lenguas en el aula implica que los profesores no solo manejen eficientemente ambas lenguas, sino que además conozcan las estructuras de la interlengua, de manera que las tengan presentes al revisar los textos escritos por los estudiantes (Svartholm, 2008). Los profesores necesitan tener conocimientos sobre los errores que tienden a cometer los niños y lo que estos informan acerca del nivel lingüístico alcanzado por estos; así también es necesario que conozcan sobre el proceso de aprendizaje de una segunda lengua.

\subsection{CONDICIONES/REQUISITOS PARA SEGUIR AVANZANDO \\ EN LA IMPLEMENTACIÓN DE UNA EDUCACIÓN BILINGÜE PARA EL SORDO EN CHILE}

Como mencionábamos más arriba, si bien la educación bilingüe para el sordo ya existe en Chile, aún queda bastante camino por recorrer para acercarse a modelos como el desarrollado en Suecia.

Uno de los aspectos cruciales para que esto sea posible es lograr un conocimiento más profundo de la LS por parte de todos los integrantes de la comunidad educativa (Humphries y Allen, 2008). Esto implica, entre otras cosas, un mayor énfasis en el desarrollo de competencias en LS en la formación inicial de los profesores de sordos; oportunidades para profundizar en el conocimiento de esta lengua una vez que los docentes están en ejercicio; políticas que logren un mayor acercamiento por parte de los padres al aprendizaje de esta lengua y la estimulación temprana de los bebés y preescolares sordos para que adquieran un buen desarrollo de la LS lo antes posible (Kushalnagar et al., 2010). La formación de profesores debiera apuntar, no solo al desarrollo de competencias en el uso efectivo de la LS, sino también al logro de un conocimiento profundo de esta - así como del castellano - que les permita reflexionar acerca de las características de ambas lenguas y establecer comparaciones. Además, es importante desarrollar en los profesores un conocimiento teórico y práctico sobre el enfoque bilingüe. La necesidad de los profesores de sordos de contar con más herramientas metodológicas para enseñar la lengua escrita a sus alumnos ha sido reportada en estudios anteriores en nuestro país (Lissi et al., 2001; Lissi et al., 2010).

Uno de los pilares de un enfoque bilingüe para el sordo, es el desarrollo lo más tempranamente posible, de la LS como primera lengua. Para lograr este objetivo se requiere, entre otras cosas, un diagnóstico temprano de la sordera en el niño y políticas y programas que apunten al aprendizaje de la LS por parte de los padres. Considerando que la mayoría de los niños sordos ingresa al sistema escolar sin un dominio del español ni de la LS, el énfasis durante los años de educación preescolar debiera estar puesto en el desarrollo de la LS y no en lograr el aprendizaje de aspectos formales de la lengua escrita. La exposición al castellano, debiera hacerse principalmente a través de la lectura compartida, en la que el profesor lee a los niños, en LS, textos auténticos que resulten significativos para estos.

Ligado a lo anterior está el rol que cumplen los adultos sordos en la educación de estos estudiantes (Mugnier, 2006). Es necesario continuar incrementando la participación de estos en número y en las responsabilidades que se les otorgan en el proceso educativo. Hasta ahora, la función principal que han cumplido las personas sordas en las escuelas ha sido el servir como modelos lingüísticos o instructores de LSCh, sin que cumplan un rol en la enseñanza de la lengua escrita. El trabajo colaborativo entre un adulto sordo 
y un profesor oyente tiene el potencial de enriquecer el proceso de aprendizaje de la segunda lengua para los estudiantes sordos. Para esto, es importante que los adultos sordos se formen también para poder trabajar el modelo bilingüe, y el contar con más profesores que sean sordos.

Vemos, entonces, que la implementación de la educación bilingüe contribuye a un desarrollo pleno de los niños y jóvenes sordos, al potenciar el desarrollo de la LS como primera lengua y al permitir usarla para facilitar el proceso de enseñanza-aprendizaje del castellano como segunda lengua, básicamente a través del uso de la lengua escrita. Sin embargo, hemos visto también que un enfoque bilingüe requiere de una serie de condiciones, muchas de las cuales están aun en un estado incipiente de desarrollo en nuestro país. Esperamos que este artículo constituya un aporte en términos de señalar algunas de estas condiciones y contribuya al debate respecto a la mejor manera de abordar la educación de los niños y jóvenes sordos. Sin embargo, es necesario continuar desarrollando estudios empíricos a nivel nacional, que ayuden a precisar donde están nuestras falencias y cómo abordarlas.

El sistema educacional chileno está impulsando cada vez con más fuerza la integración de los niños con discapacidad a la escuela regular. Debe cautelarse que esto no atente contra el derecho de los niños sordos a tener pleno acceso a la LSCh y a poder recibir instrucción en esta lengua, ya que esto podría poner a este grupo de estudiantes en una situación de inequidad, limitando sus posibilidades de una plena participación en el proceso de enseñanza-aprendizaje. Esta es una de las paradojas del movimiento hacia la inclusión, el que muchas veces parece estar más centrado en el dónde son educados los niños, que en la calidad del proceso educativo. De acuerdo a los planteamientos desarrollados en este artículo, un sistema educativo realmente inclusivo para los sordos debe ofrecer la posibilidad de una educación bilingüe para ellos.

\section{REFERENCIAS BIBLIOGRÁFICAS}

Ahlgren, I. (1984). Döva barn och skriven svenska. Forskning om teckenspråk XIII. Stockholms Universitet, Institutionen för lingvistik.

Acuña, X., Adamo, D., Cabrera, I., y Lattapiat, P. (1999). Verbos espaciales locativos en LS Chilena. Revista de Lingüística Teórica y Aplicada, vol.37, 7-22.

Adamo, D., Acuña, X., Cabrera, I. y Cárdenas, A. (1997). ¿Por qué una educación bilingüe bicultural para las personas sordas? Revista Universidad Metropolitana de Ciencias de la Educación, vol.3, 43-52.

, Cabrera, I. y Acuña, X. (2008). La educación bilingüe para las personas sordas en Chile. Ponencia presentada en el Encuentro Internacional de Investigación en Lingüística de las LS, Bogotá, Colombia, 22-24 de Octubre 2008.

, Cabrera, I., Acuña, X. y Lattapiat, P. (2003). Los clasificadores en la LS Chilena. En

M. Simón, V. Buscaglia y M. I. Massone (Comps.), Educación de sordos: Educación especial y/o educación (pp. 82-93). Editorial LibrosEnRed, www.librosenred.com.

Cabrera, I., Lattapiat, P. y Acuña, X. (1999). Verbos de concordancia en la LS Chilena. Onomázein, n.4, 335-344.

Amcoff, S. (1973). Relationer mellan språkliga uttrycksformer. Pedagogisk forskning. Uppsala: Uppsala universitet, Lärarhögskolan. 
Bergman, B. (1977). Tecknad svenska. SÖ rapport FoU 28. Stockholm: Liber (Traducido al inglés en 1979: Signed Swedish. Swedish National Board of Education. Liber: Stockholm). (1979). Dövas teckenspråk - en inledning. [Lengua de señas de las personas sordas - una introducción]. Stockholm: Forskning om Teckenspråk III. Stockholms universitet, Institutionen för lingvistik.

(1982). Några satstyper i det svenska teckenspråket. [Algunos tipos de oraciones en Lengua de Señas Sueca]. Stockholm: Forskning om Teckenspråk XI. Stockholms universitet, Institutionen för lingvistik. (1990). Grammaticalization of location. In W.M Edmondson y F. Karlsson, SLR'87. Papers from the Fourth International Symposium on Sign Language Research. Lappeenranta, Finland July 15-19, 37-56.

(1994). Signed languages. In I. Ahlgren y K. Hyltenstam (Eds.), Bilingualism in Deaf Education (pp. 15-36). Hamburg: SIGNUM Press.

Erting, C. (1988). Acquiring linguistic and social identity: Interactions of deaf children with a hearing teacher and a deaf adult. In M. STRONG (Ed.) Language Learning and Deafness. (192-219). Cambridge University Press: Cambridge.

Gibbons, P. (2002). Scaffolding language scaffolding learning. Portsmouth: Heinemann.

Gibson, H., Small, A. Y Mason, D. (1997). Deaf bilingual bicultural education. In J. Cummins y D. Corson (Eds.), Encyclopedia of Language and Education, Vol. 5: Bilingual Education (231-241). London: Kluwer Academic Publishers.

Halliday, M. A. (1985). An introduction to functional grammar. London: Edward Arnold.

Hendar, O. (2008). Måluppfyllelse för döva och hörselskadade $i$ skolan. [Metas logradas por los estudiantes sordos e hipoacúsicos en la ëscuela.] Örebro: SPM.

Hermans, D., Knoors, H., Ormel, E., y Verhoeven, L. (2008). Modeling reading vocabulary learning in deaf children in bilingual education programs. Journal of Deaf Studies and Deaf Education, vol.13, n.2, 155-174.

Herrera, V. (2010). Estudio de la población sorda en Chile: evolución histórica y perspectivas lingüísticas, educativas y sociales. Revista Latinoamericana de Educación Inclusiva, vol.4, n.1, 211-226.

Hugounenq, H. (2006). Le statut politique de la langue des signes éléments de réflexion sur la perception du bilinguisme en France. Marges Linguistiques, n.10, 1-16.

Humphries, T. y Allen, B. (2008). Reorganizing teacher preparation in deaf education. Sign Language Studies, vol.8, n.2, 160-183.

Johnson, R. E., Liddell, S. K. y Erting, C. J. (1989). Unlocking the currículum: Principles for achieving access in deaf education. Gallaudet Research Institute Working Paper 89-3. Washington, D.C: Gallaudet University.

Kushalnagar P., Mathur G., Moreland, C.J., Napoli, D.J., Osterling, W., Padden C., Rathmann C. (2010). Infants and children with hearing loss need early language access. Journal of Clinical Ethics, vol.21, n.2, 143-154.

Lasasso, C. (2000). National survey of bilingual-bicultural programs in the United States and implications for bilingual-bicultural research in the United States and other countries. Paper presented at the 19th International Congress on Education of the Deaf and 7th Asia-Pacific Congress on Deafness, July 12, 2000, Sydney, Australia (Unpublished).

. y J. Lollis, J. (2003). Survey of residential and day schools for deaf students in the United States that identify themselves as bilingual-bicultural programs. Journal of Deaf Studies and Deaf Education, vol.8, n.1, 79-91.

Ley 20.422 (2010). Establece normas sobre igualdad de oportunidades e inclusión social de personas con discapacidad. Consultado el 20 de noviembre 2010 en: http://www.senadis.cl/centro/ ley_20422.php 
LGr80 (1983). Supplement till LGr80, Läroplan för specialskolan. [Suplemento de LGr80, Currículum para la Escuela Especial, Orientaciones Adicionales]. Stockholm: Skolöverstyrelsen, Liber.

Liddell, S. K. (2003). Grammar, gesture and meaning in American Sign Language. Cambridge: Cambridge University Press.

, Vogt-Svendsen, M. y Bergman, B. (2007). A crosslinguistic comparison of buoys: Evidence from American, Norwegian, and Swedish Sign Language. In M. Vermeerbergen, L. Leesen y O. Crasborn (Eds.), Simultaneity in Signed Languages (pp. 187-215).: Amsterdam/ Philadelphia: John Benjamins Publishing Company.

Lissi, M. R., Cabrera, I., Raglianti, M., Grau, V. y Salinas, M. (2003). Literacidad en escolares sordos chilenos: Evaluación y desafíos para la investigación y la educación. Psykhe, vol.12, n.2, 37-50.

, Grau, V., Raglianti, M., Salinas, M. Y Torres, M. (2001). Adquisición de la lectoescritura en niños sordos: Una visión desde los profesores en Chile. Psykhé, vol10, n.1, 35-48.

, Salinas, M., Acuña, X., Adamo, D., Cabrera, I., y González, M. (2010) Using Sign Language to teach written language: an Analysis of the strategies used by teachers of deaf children in a bilingual context. L1 - Educational Studies in Language and Literature, vol.10, n.1, 57-69.

Lpo 94 (1994). Läroplan för det obligatoriska skolväsendet, förskoleklassen och fritidshemmet 1994. [Currículum para la escuela obligatoria, el aula preescolar y el centro y programas para después de la escuela.] Stockholm: Utbildningsdepartementet.

Lynas, W. (2005). Controversies in the education of deaf children. Current Pediatrics, vol.15, n.3, 200-206.

Mahshie, S. N. (1995). Educating deaf children bilingually with insights and applications from Sweden and Denmark. Washington, DC: Gallaudet University, Pre-College Programs.

Marchesi, A. (1987). El desarrollo cognitivo y lingüístico de los niños sordos. Perspectivas educativas. Madrid: Alianza Editorial.

(2001). Desarrollo y educación de los niños sordos. En A. Marchesi, C. Coll y J. Palacios (Comp.), Desarrollo psicológico y educación. Vol. 3: Trastornos del desarrollo y necesidades educativas especiales (pp. 241-271). Madrid: Alianza Editorial.

Marschark, M., Lang, H. G. y Albertini, J. A. (2002). Educating Deaf Students. From Research to Practice. Oxford: Oxford University Press.

Mather, S. A. (1987). Eye gaze y communication in a deaf classroom. Sign Language Studies, vol.54, n.1, 11-30.

Miranda, M. (1997). Evaluación de la comprensión lectora en alumnos sordos. Tesis para optar al grado de Magister en Educación diferencial, UMCE, Santiago, Chile.

Moores, D. (1987). Educating the deaf. Psychology, principles, and practices. Boston: Houghton Mifflin Company.

Mugnier, S. (2006). Le bilinguisme des enfants sourds: de quelques freins aux posibles moteurs. Glottopol, n.7, 144-159.

Nordén, K (1969). Begåvningsstrukturen hos en grupp döva ungdomar. Rapport no. 0014. Stockholm: Personaladministrativa Rådet.

Oviedo, A. (2008). Algunos documentos acerca de la historia de la educación de sordos en Chile (contenidos en los Anales de la Universidad de Chile). Consultado el 14 de septiembre de 2010 en: http://www.cultura-sorda.eu/resources/Oviedo_escuelas_sordos_Chile_2008.pdf

Palincsar, A. S. y Brown, A. L. (1984). Reciprocal Teaching of comprehension-fostering and comprehension-monitoring activities. Cognition and Instruction, vol.1, n.2, 117-175.

Paul, P. V. y Quigley, S. P. (1994). Language and deafness. San Diego, CA: Singular Publishing Group.

Power, D y G. Leigh (2000). Principles and practices of literacy development for deaf learners: A historical overview. Journal of Deaf Studies and Deaf Education, vol.5, n.1, 3-8. 
Schembri, A. (2003). Rethinking "classifiers" in signed languages. En K. Emmorey, Perspective on classifier constructions in sign languages (3-34). New York: Psychology Press.

Schirmer, B. (2000). Language and literacy development in children who are deaf. Boston, MA: Allyn y Bacon.

SPM (2001, 2002, 2005, 2007). Årsrapporter. Örebro: National Agency for Special Schools for the Deaf and Hard of Hearing.

SPSM (2008). Årsrapport. Härnösand: National Agency for Special Needs Education and Schools.

Strong, M (1995). A review of bilingual-bicultural programs for deaf children in North America. American Annals of the Deaf, vol.140, n.2, 84-94.

Svartholm, K. (1984). Döva och samhällets skrivna språk. Forskning om teckenspråk XII. Stockholms universitet, Institutionen för lingvistik: Stockholm.

(1987a). Subjekt och objekt i dövas skrivna svenska. [Sujeto y objeto en el lenguaje escrito del sordo] In E. Wande et al., Aspects of Bilingualism. Proceedings from the Fourth Nordic Symposium on Bilingualism, (pp. 419-435). Uppsala: Acta Universi-tatis Upsaliensis, Studia Multiethnica Upsaliensia. (1987b). Relativsatser och andra konstruktioner i dövas skrivna svenska. [Cláusulas relativas y otros constructos en el sueco escrito del sordo.] MINS 24. Stockholms universitet, Institutionen för nordiska språk.

(1988). Uttryck för befintlighet och förflyttning i dövas skrivna svenska. [Expresiones indicando lugar y movimiento el sueco escrito del sordo] In K. Hyltenstam y I. Lindberg, Första symposiet om svenska som and $\neg$ raspråk [Primer Simposio sobre Sueco como Segunda Lengua] Vol. I. (pp. 234-245). Stock $\neg$ holms universitet, Centrum för två $\neg$ språkighetsforskning.

. (1993). Bilingual education for the deaf in Sweden. Sign Language Studies, vol.81, 291-332.

(1997). Döva barns tvåspråkighet. [Bilingualism for deaf children.] In G. Bredberg y S. Harris, Vårdprogram för behandling av döva barn med cochleaimplantat. [Care program for treatment of deaf children with cochlear implants.] (pp. 27-31). Stockholm y Lund. (1998). Bilingual education for the deaf: Evaluation of the Swedish model. En: G. L. Zaitseva, A. A. Komarova y D. M. Pursglove, Deaf children and bilingual education. Proceedings of the International Conference on Bilingual Education of Deaf Children, Moscow, April 1996. (pp. 136-146). Moscow: Zagrey.

(2007). Cochlear-implanted children in Sweden's bilingual schools. In L. Komesaroff (Ed.), Surgical consent. Bioethics and cochlear implantation (pp. 137-49). Washington, D.C.: Gallaudet University Press.

. (2008): The written Swedish of deaf children: A foundation for EFL. In C. J. Kellett Bidoli y E. Ochse, English in International Deaf Communication (pp. 211-249). Bern: Peter Lang.

. (2010). Bilingual education for deaf children in Sweden. International Journal of Bilingual Education and Bilingualism, vol.13, n.2, 159-174.

Andersson, R. y Lindahl, U. (1993). Samspråk i dövundervisning. Studier av klassrumskommunikation i två olika skolformer för döva. Stockholm: Stockholms universitet, Institutionen för nordiska språk.

Vygotsky, L. S. (1962). Thought and language. Massachusetts: M.I.T. Press.

Wilbur, R. (2000). The use of ASL to support the development of English and literacy. Journal of Deaf Studies and Deaf Education, vol.5, n.1, 81-104. 